\title{
Le sens des transformations et les transformations du sens : les changements du sens de l'activité à l'échelle biographique
}

\begin{abstract}
Abstrait
Cet article porte sur la manière dont se structurent les transformations du sens de l'activité chez les dirigeants des entreprises de l'économie sociale (coopératives, mutuelles, associations et fondations) à l'occasion des événements marquants vécus le long de leur histoire professionnelle. Cinq éléments structurant les transformations du sens sont présentés : la dimension temporelle ; les émotions associées ; la reconnaissance d'un changement par le sujet lui-même ; l'énoncé de principes d'action associés aux transformations et l'imbrication des dimensions individuelle et collective. Par ailleurs, la lecture diachronique des transformations permet d'identifier le sens ou direction attribuée par les dirigeants à leur propre parcours. Ces résultats, obtenus dans le cadre d'une recherche qualitative par l'analyse narratologique (Labov) des récits produits par treize dirigeants d'entreprises de l'économie sociale lors d'entretiens non-directifs, caractérisent les transformations du sens et par conséquent son processus de construction et de reconstruction dans une perspective diachronique.
\end{abstract}

Mots-clés : construction de sens, dirigeants, événements marquants, activité professionnelle, formation.

\section{The Meaning of Transformations and Transformations of Meaning: Changes in the Meaning of Activity at the Biographical Scale}

\footnotetext{
Abstract

* Conservatoire National des Arts et Métiers, Paris, France.

Article soumis le 28 février 2021 ; accepté pour publication le 9 mai 2021.
}

This article focuses on the way in which transformations of the meaning of activity among CEOs of companies of the social economy movements (cooperatives, mutual societies, associations and foundations), are structured by the significant events 
experienced throughout their professional history. Five elements structuring the transformations of meaning are presented: the temporal dimension; emotions; the recognition of a change by the subject himself; the statement of principles of action associated with transformations and the interweaving of individual and collective dimensions. Moreover, the diachronic reading of the transformations makes it possible to identify the meaning or direction attributed by the leaders to their own paths. These results, obtained through qualitative research by the narrative analysis (Labov) of the accounts produced by thirteen CEOs during non-directive interviews, characterize the transformations of meaning and subsequently the process of construction and reconstruction from a diachronic perspective.

Keywords: construction of meaning, CEOs, social economy, significant events, professional activity.

\section{Introduction}

Notre activité transforme notre environnement tout autant qu'elle nous transforme, sans que nous en soyons toujours conscients. Une activité mentale et affective permanente, désignée ici comme processus de construction de sens (Barbier 2017), accompagne et oriente notre activité quotidienne.

L'activité professionnelle constitue un champ riche pour l'étude de la construction de sens car elle articule de manière complexe plusieurs dimensions : la dimension individuelle et collective du travail, ses contraintes organisationnelles ; sa dimension culturelle ; les enjeux de production ; les écarts entre le prescrit et le réel, entre le prévu et le réalisé... Nous explorerons dans cet article le processus de transformation du sens portant sur l'activité professionnelle et caractériserons les éléments structurant ses transformations.

Pour analyser ce processus, nous partons de l'idée que certaines situations professionnelles ${ }^{1}$, parce qu'inédites, ouvrent au chercheur un accès privilégié aux transformations du sens. Contrairement à l'activité mentale accompagnant les situations de routine ${ }^{2}$, les situations inédites, par la rupture qu'elles instaurent dans le flux réflexif du sujet, demandent de lui une réflexion intense. Nous nous intéressons ainsi dans ce texte au processus de transformation de sens portant sur l'activité professionnelle à l'occasion des expériences épisodiques (Rogalski, Leplat 2011), désignées comme événements marquants. Ces expériences singulières constituent des jalons dans la vie professionnelle transformant le sens attribué par

\footnotetext{
1 La situation professionnelle est définie ici comme « la qualification par un sujet du contexte dans lequel il agit » (Barbier 2017).

2 Rogalski et Leplat (2011) distinguent les expériences sédimentées des expériences épisodiques. La première modalité met l'accent sur ce qui est acquis comme effet de la répétition des tâches lors de la pratique professionnelle, tandis que la deuxième renvoie à des situations singulières contextualisées et liées à l'ici et maintenant du travail.
} 
les sujets à leur activité. L'analyse des récits portant sur les événements marquants vécus par des dirigeants d'entreprises de l'économie sociale en France ${ }^{3}$ rend possible non seulement l'appréhension de la perspective diachronique du développement de l'activité professionnelle mais révèle également « le sens des transformations » singulières dans un empan temporel long. Le sens des transformations fait référence à la direction que les dirigeants attribuent à leur histoire professionnelle sous-tendant une causalité chronologique qui justifie et légitime in fine leur statut.

Plusieurs éléments font des dirigeants des entreprises de l'économie sociale un terrain fertile pour l'analyse des transformations du sens.

Dans les organisations, les dirigeants sont investis d'un pouvoir de réalisation d'objectifs et d'organisation de moyens pour le faire (Barbier et all. 2011). De leur position statutaire particulière découle la prise de décisions définissant des engagements à long terme pour l'organisation (Mintzberg 1990). En somme, le défi de tout dirigeant consiste à faire que les missions et finalités de l'organisation soient réalisées en donnant du sens à la production collective (Mintzberg 1984). Pour les dirigeants des entreprises de l'économie sociale ce défi est double car leur appartenance à un mouvement d'idées et d'initiatives économiques caractérisé par un discours sur les valeurs (solidarité, générosité, responsabilité, engagement...) les contraint à un " travail du sens » sur deux dimensions (Rodriguez 2017). La première consiste à orienter le sens construit par les collaborateurs sur leurs activités et les enjeux collectifs, en explicitant les buts et les finalités de l'organisation. La deuxième consiste à inscrire le sens des actions collectives et des décisions stratégiques dans l'ensemble de valeurs prônées par le mouvement de l'économie sociale. Cela s'effectue principalement par la référence à l'intérêt général (Mispelblom Beyer 2010).

Diriger une organisation est une activité dont les tâches sont faiblement prescrites (Leplat, Hoc 1983) ${ }^{4}$ et pour laquelle il n'existe pas de formation formelle ${ }^{5}$. Ce dernier élément est d'autant plus significatif pour les entreprises de l'économie sociale que la plupart des dirigeants évoluent souvent dans la hiérarchie

\footnotetext{
${ }^{3}$ L'économie sociale et solidaire (ESS) désigne actuellement en France un ensemble d'entreprises organisées sous forme de coopératives, mutuelles, associations, ou fondations, dont le fonctionnement interne et les activités sont fondés sur un principe de solidarité et d'utilité sociale. Leurs modes de gestion se veulent démocratiques et participatifs. (Ministère d'Economie, des finances et de la relance).

${ }^{4}$ La tâche prescrite, selon Leplat et Hoc, « c'est la tâche conçue par celui qui en commande l'exécution » (1983: 52).

5 Il existe des formations spécifiques portant sur des techniques de gestion ou d'administration qui ne s'adressent pas exclusivement aux dirigeants. Lors de nos travaux de recherche, nous avons participé à la conception de dispositifs de formation à destination de personnes dites " à haut potentiel ", des personnes identifiées dans les organisations comme susceptibles de prendre des responsabilités dans un futur plus ou moins proche. Les participants s'heurtaient néanmoins à la difficulté d'imaginer une activité complexe qu'ils n'exerçaient pas encore.
} 
grâce à leur fort engagement militant (Apec 2012) où la place occupée par l'organisation dans leur histoire personnelle et familiale n'est pas négligeable ${ }^{6}$.

Nous faisons l'hypothèse que ces éléments ont une forte incidence sur la nécessité chez les dirigeants d'apprendre « sur le tas » pour réussir leurs missions.

Les résultats et réflexions présentés dans ce texte sont issus d'une recherche qualitative en Sciences de l'éducation (Rodriguez 2017), dont l'objectif était d'explorer les caractéristiques des constructions de sens chez les dirigeants de l'économie sociale dans une démarche d'intelligibilité (Barbier 2000). L'une des principales hypothèses posées dans le cadre de la recherche, considérait que le processus de formation à l'activité professionnelle sur le long terme peut être analysé par le biais des constructions des sens relatives à cette activité. Les récits narratifs portant sur les événements marquants constituent, de ce point de vue, un moyen nous permettant d'accéder aux traces des constructions de sens survenues dans le cadre de l'expérience professionnelle. Nos analyses ne portent donc pas sur le contenu des activités des dirigeants ou leur pertinence mais sur la manière dont ceux-ci les évoquent et les perçoivent. L'économie sociale constitue pour sa part un contexte pertinent pour ce type d'analyse au regard de la place occupée par les valeurs et par conséquent au sens de l'activité économique et sociale des entreprises dans les discours. Plutôt que chercher à évaluer la cohérence entre les actions rapportées par les dirigeants dans leurs récits et la philosophie ou la démarche économique et sociale de l'économie sociale, nous nous sommes centrée sur la manière dont cette philosophie modèle les constructions de sens des dirigeants.

Dans cet article, nous présenterons d'abord la notion de sens et ses liens avec l'activité humaine ainsi que les caractéristiques et la fonction des événements marquants comme vecteurs de transformation. Nous exposerons par la suite le cadre méthodologique de la recherche et les principales caractéristiques du mouvement de l'économie sociale et de l'activité de ses dirigeants. Nous finirons par décrire les éléments impliqués dans les transformations de sens des dirigeants de l'économie sociale : la dimension temporelle ; les émotions qui leur sont associées ; la reconnaissance du changement par le sujet ; l'énoncé des principes d'action et l'imbrication de la dimension individuelle et collective.

\footnotetext{
${ }^{6}$ Il est fréquent d'ailleurs de rencontrer des dirigeants ayant commencé leur vie professionnelle dans la même structure qu'ils dirigent. Depuis une dizaine d'années, ce modèle de recrutement commence à perdre son élan au profit d'un modèle privilégiant les qualifications, ce qui fait l'objet de réactions de la part des militants.
} 


\section{Sens, activité et événements marquants}

\section{La construction de sens comme processus de symbolisation}

Le sens est considéré par la psychologie comme la résultante d'une interaction entre un élément fonctionnant comme symbole et l'expérience pré-conceptuelle du sujet (Gendlin 1962). L'expérience pré-conceptuelle correspond au fonctionnement continu du sujet, c'est-à-dire, au flux présent de ses sensations et de ses perceptions. Le sujet construit du sens grâce à un regard vers l'intérieur, vers la masse toujours présente de sensations. Le sens serait ainsi la résultante d'un travail de symbolisation à laquelle le sujet accède par l'introspection, et sa construction, un processus de symbolisation à partir de son expérience. La symbolisation évoquée par Gendlin (1962) se réalise par le langage, système symbolique partagé et médiation culturelle fournissant au sujet une représentation du monde. Pour Jerome S. Bruner (1990), avant même l'acquisition du langage chez le sujet, il existerait du sens sous la forme de représentations proto-linguistiques du monde, dont la réalisation dépend de l'outil culturel du langage.

\section{L'organisation narrative du sens}

La psychologie narrative postule que la structure psychologique humaine est essentiellement narrative et que la narration constitue un principe d'organisation de l'activité (Sarbin 1986, cité par Crossley 2000 ; Bruner 1991). Nous donnons forme à notre expérience, nous construisons du sens, selon une structure narrative. L'être humain viendrait au monde avec un ensemble de prédispositions pour interpréter l'espace social et pour agir car «nous avons une prédisposition innée et primitive à l'organisation narrative qui nous permet très vite de comprendre et de l'utiliser » (Bruner 1991 : 92). La structure narrative agirait comme une sorte de matrice déterminant un mode de fonctionnement cognitif particulier ${ }^{7}$ (Bruner 1991). Ce mode cognitif narratif place l'expérience humaine, c'est-à-dire l'intention et l'action, dans un temps et dans un espace. Notre expérience s'organiserait donc selon des formes narratives dont la dimension temporelle est l'une des composantes : " notre expérience assume de manière automatique des formes temporellement déployées dans lesquelles le futur, le passé et le présent se déterminent mutuellement comme des parties d'un ensemble » (Crossley 2000 : 37).

En construisant du sens, processus de symbolisation prenant une forme narrative, nous organisons les épisodes, les actions et les récits relatifs à nos actions. Ce processus de symbolisation à la fois organise notre expérience et donne forme à nos actions.

\footnotetext{
${ }^{7}$ Bruner (1991) distingue le mode de fonctionnement cognitif narratif du mode paradigmatique dont le principe organisateur est le système formel de description et d'explication de la réalité empirique qu'il a pour but de vérifier.
} 
Quant au champ spécifique de l'activité professionnelle, il est intéressant de noter que pour Clot (2004), le sens est un ressort interne de l'activité de travail défini comme une "discordance créatrice ou destructrice entre les occupations et les pré-occupations du sujet » (ibidem : 325). Les occupations concernent l'activité réelle du sujet, tandis que les pré-occupations sont « les conflits vitaux de l'activité, les soucis, les mobiles personnels et collectifs » (ibidem) présents dans la réalisation du travail. Les pré-occupations (Clot 2004) définissant et définies par l'activité du sujet, contiennent entre autres : l'activité d'autrui, l'organisation du travail, les conflits, le regard d'autrui vis-à-vis de son activité, par exemple qui constituent également des composantes des trames narratives du sens.

En somme, trois caractéristiques définissent ici le sens et sa construction : le sens est en même temps un processus de symbolisation et sa résultante, produit par la médiation du langage et prenant une structure narrative. En outre, la construction de sens en tant qu'activité de symbolisation, non seulement accompagne mais façonne aussi l'action du sujet en prenant appui sur les trames narratives développées. La solidarité est donc forte entre l'expérience directe du sujet (ou l'activité réelle selon Clot 2004) et l'activité de construction de sens car elles se nourrissent et se transforment mutuellement. Dans le contexte spécifique de l'activité professionnelle, la construction de sens se voit affectée par la perception que le sujet a de l'activité d'autrui et de l'organisation du travail, entre autres.

\section{Les événements marquants comme vecteurs de construction de sens}

Mais dans le flux permanent de notre activité nous retrouvons les situations courantes, configurant nos expériences sédimentées et les situations constituant des épisodes à caractère exceptionnel, autrement dit les expériences épisodiques (Rogalski, Leplat 2011).

Les événements marquants, uniques et singuliers, adviennent au sujet et constituent pour lui une rupture dans le flux de son expérience, devenant des expériences épisodiques. Les événements, parce qu'inattendus, désorganisent les habitudes de perception créant pour le sujet une situation confuse, incertaine et imprévisible qui ouvre la voie à un processus de réflexion, à des questions, et à une quête de réponses (Dewey 1993 ; Leclerc-Olive 1997).

La notion d'événement contient à la fois la notion de ce qui advient (l'imprévu) et la manière dont le sujet l'accueille. L'interprétation de ce type d'expériences par le sujet s'effectue dans la tension qui lie les expériences passées aux expectations, attentes, projets et représentations de l'avenir (Leclerc-Olive 1997).

L'espace ouvert par l'événement déclenche un processus de transformation de sens chez le sujet rendant possible la compréhension et l'action dans une situation perçue par lui comme inédite et a priori désorganisée. 


\section{Les transformations de sens sur l'activité des dirigeants produites par le vécu d'événements marquants}

\section{Les principales caractéristiques du mouvement et de l'activité des dirigeants de l'économie sociale}

Le mouvement de l'économie sociale en France se caractérise par les discours tenus par ses membres à propos de la cohérence entre des valeurs historiquement affichées et les pratiques socio-économiques qu'elles accompagnent. Le jeu entre valeurs affichées et pratiques économiques a lieu dans des structures dont les statuts juridiques traditionnels sont ceux des associations, mutuelles et coopératives appelées souvent « familles ». Leur fonctionnement est régi par des principes considérés par le mouvement comme s'opposant à ceux des entreprises dites capitalistiques : la libre adhésion ; la participation des adhérents à la prise des décisions qui se veut démocratique (une personne une voix) ; la cotisation et la part sociale (respectivement droit d'accès aux services et titre de propriété sur une part du patrimoine de l'entreprise) ; la non-lucrativité (les réserves de l'entreprise associative sont impartageables); des bénéfices réinvestis dans l'entreprise afin de maintenir et développer son activité ; des statuts non immuables (Demoustier 2001 ; Jeantet 2004). Le trait commun des entreprises réunies sous l'appellation " économie sociale ", serait leur " adhésion commune à la démocratie et à la primauté de l'objectif social sur la recherche du profit » (Robert 2007 : 15) visant l'émancipation (Draperi 2009).

Si les valeurs et finalités du mouvement invitent à y adhérer, il n'en reste pas moins vrai que leur mise en œuvre semble difficile. Or, c'est sur les dirigeants de ces structures que repose en grande partie la responsabilité de trouver des voies concrètes à la réalisation de ces vœux.

Outre la question du sens, l'activité de ces dirigeants consiste à gérer la tension entre l'équilibre financier de l'organisation, la réponse aux enjeux de développement et de concurrence des marchés et le respect du cadre juridique et financier, sans (trop) contrevenir aux valeurs enoncées par le mouvement.

La particularité à diriger des structures de l'économie sociale peut être résumée en trois éléments :

- l'appartenance de l'organisation à un regroupement, motivé principalement par une démarche de reconnaissance de sa spécificité auprès des pouvoirs publics. En effet, depuis une cinquantaine d'années, le mouvement de l'économie sociale en France vise à faire reconnaître ce que ses entreprises apportent à la société en termes de contribution à garantir l'intérêt général dans la prise en charge par exemple des publics dits fragiles ;

- un discours axé sur le sens même de l'activité de ces entreprises, leur utilité sociale et leur démarche démocratique par rapport aux entreprises dites 
capitalistiques dont le but principal serait de produire des bénéfices pour les détenteurs du capital ;

- l'activité des dirigeants comme la gestion de la tension entre les valeurs dont le mouvement est porteur, et les injonctions propres au marché (lois de respect de la concurrence, besoin de produire des bénéfices...) ( Betton et all. 2008).

\section{Le cadre méthodologique de la recherche}

Des entretiens non directifs portant sur le parcours professionnel, menés auprès d'un échantillon de treize dirigeants d'entreprises de l'économie sociale 8 âgés d'entre 28 et 68 ans, ont donné lieu à la production de 39 récits d'expérience. Chaque dirigeant a évoqué en moyenne trois événements.

La seule consigne donnée a été de raconter les événements restés dans la mémoire des interviewés parce que significatifs pour leur activité de dirigeants. Les dirigeants ont fait eux-mêmes le choix des événements considérés dans l'ici et maintenant de l'entretien comme marquants.

Les entretiens non directifs ont fait appel de manière implicite à la mémoire épisodique (Tulving 1983, cité par Nicolas 2005) ${ }^{9}$ des interviewés. Cette technique de recueil des données part du postulat que le degré de difficulté rencontré par un sujet lors de la résolution d'un problème constitue l'un des facteurs de mémorisation d'une situation. La difficulté à comprendre et à résoudre la situation seraient ainsi des facteurs responsables d'une mémorisation privilégiée chez le sujet, capable de restituer le souvenir a posteriori de manière complète.

Le travail de mise en récit des événements du passé a sollicité d'une part les constructions de sens produites au moment même du vécu des événements et gardées en mémoire, en même temps que des productions ayant lieu dans l'ici et maintenant de l'entretien. La posture d'évocation chez les dirigeants a été facilitée par la demande de raconter des situations concrètes plutôt que des avis généraux sur l'activité de diriger.

La méthode d'analyse des données a consisté dans l'identification et l'analyse des composantes narratologiques des récits portant sur les événements marquants passés, mais produits dans le cadre de l'entretien non-directif. Les récits contiennent des traces narratives propres aux constructions de sens passées ainsi que des constructions nouvelles, émergeant dans le cadre de l'entretien. Les postulats de la psychologie narrative permettent de faire l'hypothèse que ces récits n'ont pas été créés ex-nihilo dans le cadre de l'entretien mais que, au contraire, ils portent les traces des récits construits par le passé. Pour distinguer les traces des récits

\footnotetext{
8 Six associations, cinq coopératives, une mutuelle et une fondation.

9 Celle-ci est définie comme « un système qui reçoit et stocke l'information concernant des épisodes ou des événements temporellement datés ainsi que les relations spatio-temporelles entre ces événements » (Nicolas 2005 : 388).
} 
antérieurs, les outils proposés par la narratologie, et notamment ceux développés par William Labov $(1993,2003,2010)$ permettent d'identifier les évaluations des événements par le sujet et les rationalisations effectués dans le présent de l'entretien et portant sur les événements ou les actions du passé, par exemple. Souvent les dirigeants précisaient spontanément si leurs réflexions étaient nouvelles ou celles qu'ils avaient eues à l'époque.

L'analyse des entretiens a consisté à isoler les récits narratifs, définis selon Labov (1993) par le fait que les propositions y sont ordonnées temporellement, de sorte que toute inversion modifie l'ordre des événements tel que l'on peut l'interpréter. Ensuite, pour chaque narration portant sur un événement, et par syntagme, les séquences définissant la structure générale des récits d'expérience personnelle (Labov 1993, 2003, 2010) ont été identifiées : résumé, indications, développement, évaluation et conclusion. Nous avons ainsi procédé pour chaque séquence à l'identification des composantes des récits (Adam, Revaz 1996) : références temporelles (absolues, relatives à l'énoncé et celles relatives à la situation d'entretien) et spatiales (périmètre) ; les rôles attribués aux personnages (principal, adjuvant, antagoniste, destinateurs et destinataires) ${ }^{10}$; les émotions énoncées par les narrateurs ; la durée et la vitesse du récit (tension entre le racontant et le raconté11) ; les transformations (situation initiale vs situation finale) ; les actions des personnages ; l'agence (agency) des personnages (sujet opérateur ou patient) ; la perspective de la narration (focalisation interne aux personnages ou neutre et changement de focalisation) ; enchaînements causales et enchaînement chronologiques. ${ }^{12}$ Les analyses ont donné lieu à l'identification d'éléments saillants présents dans les récits portant sur les événements marquants : les liens établis entre passé, présent et futur (ils se manifestent de manière singulière dans chaque récit) ; l'identification par le narrateur d'une transformation dans la manière de percevoir leur propre fonction ; la référence aux émotions ressenties ou la survenue de ces émotions lors de l'entretien ; l'expression de ce que nous appelons des principes d'action, c'est-à-dire, des règles selon lesquelles le dirigeant considère organiser son activité et, l'imbrication des dimensions individuelle et collective dans les récits. Ces éléments sont présents dans tous les récits, mais prennent une place ou une forme singulière pour chacun.

\footnotetext{
${ }^{10}$ Ces personnages sont issus du modèle théorique développé par Algirdas, Greimas 1966 (cité par Adam, Revaz 1996).

${ }^{11}$ Le racontant est défini comme « le texte ou énoncé dans la linéarité orale » tandis que le raconté est l'histoire ou contenu narratif. (Adam, Revaz 1996).

${ }^{12}$ Des analyses complémentaires dont l'objet a été d'identifier la mise en intrigue, l'attribution implicite de responsabilités ainsi que les ruptures et continuités liées à la représentation de leur activité ont été menées.
} 


\section{Les éléments intervenant dans la transformation du sens de l'activité de diriger une entreprise de l'économie sociale à l'occasion des événements marquants}

A partir de l'analyse narratologique des récits, cinq éléments ont été identifiés comme structurant le processus de transformation du sens de ce qu'est diriger une entreprise de l'économie sociale.

\section{La dimension temporelle : l'articulation du passé, du présent et du futur présente dans les transformations du sens de diriger}

Les récits portant sur les événements marquants se structurent à partir de l'articulation entre passé, présent et futur des faits par le narrateur. Bien que cette dimension soit propre à toute narration, sa prise en compte dans l'analyse du processus de construction de sens met en lumière le rôle de celle-ci dans l'identification des transformations du sens. Autrement dit, la référence au temps permet de définir la transformation et de la qualifier comme telle, en établissant une différence d'état entre le passé, le présent et le futur.

Dans cet extrait, une dirigeante âgée de 50 ans d'une importante association nationale de protection de l'adolescence, fait la narration d'un événement marquant vécu lorsqu'elle avait 21 ans.

je dirais que le premier moment vraiment marquant c'est quand à la veille de signer un contrat avec l'université pour être assistante je n'ai pas signé mais ça a été un moment marquant pour moi parce que c'était prévu et je le souhaitais parce que tout d'un coup j'ai réalisé que j'étais entrée à l'école à deux ans et demi et que j'allais en sortir à soixante-dix ans et qu'à l'époque j'avais 21 ans et je me suis dit je n'aurais jamais rien connu d'autre j'ai eu une peur et donc j'ai dit non alors que j'irais à la fac hein c'était pas le problème mais j'étais vraiment ça m'a saisie et dans les 15 jours j'ai eu une proposition d'emploi qui m'a intéressée et que j'ai acceptée (Dirigeante 2, association).

Le récit lie l'événement, l'offre d'un poste à l'université qui l'aurait empêchée de devenir dirigeante, à son passé (son parcours à l'école depuis ses 2 ans) et son futur (sortir du système éducatif à l'âge de la retraite) vus depuis le moment de l'événement (le moment de signer l'acceptation de l'emploi à l'université). La future dirigeante se retrouve face à un dilemme qui semble définir son « destin ». Elle dit se représenter son passé de l'époque, elle a 21 ans lors de l'événement, et le futur qui l'attendait dans le cas où elle accepterait l'offre. Cette interprétation de la situation vécue à l'époque, où une décision considérée comme déterminante pour sa vie devait être prise contient donc les liens entre ces trois temps passé, présent de l'événement et futur représenté. A ces trois temps de l'événement, se rajoute le 
futur proche de l'événement, l'acceptation d'un autre poste 15 jours après. Le récit laisse voir la dimension temporelle de la transformation de sens. Bien que le poste à l'université l'attirât, l'interprétation construite à l'époque, celle d'une vie professionnelle passée au sein du système éducatif, ouvre la voie à une décision qui l'amènera des années plus tard, vers le poste de dirigeante.

\section{Les émotions associées aux transformations du sens de diriger}

Un dirigeant de coopérative de 28 ans revient lors de l'entretien sur les difficultés rencontrées au moment de la prise en charge de l'organisation. Il s'agit d'une coopérative de construction en bois située dans une région rurale de France dont les objectifs sont économiques et sociaux : l'accueil des nouveaux arrivants sur le territoire, la mise en valeur de la ressource du bois issue des forêts locales et le métier artisanal de la charpente.

En réponse à la consigne donnée dans le cadre de l'entretien, le dirigeant relate son arrivée dans l'entreprise et s'arrête sur le moment de crise le plus profond qui va l'amener à prendre la direction de la structure.

euh en 2008 on a une activité qui est euh précaire au niveau de la rentabilité des chantiers on a beaucoup perdu et d'ailleurs beaucoup de gens pensent que notre structure est est morte (pleurs) oui ça c'est ça c'est je suis ému parce que c'était dur (pleurs) et là ça a été dur parce que on s'engage dans un travail artisanal où en milieu rural où on est assez peu nombreux sur le territoire et où la notoriété la reconnaissance du travail bien fait c'est quelque chose d'important et nous on a des difficultés sur notre je l'ai déjà dit sur la rentabilité aussi sur la qualité de nos chantiers sur notre crédibilité en fait quoi et du coup moralement c'est hum (ému) c'est quand même quelque chose de:: de dur parce que on c'est là qu'on sent le décalage entre l'engagement idéologique et la réalité du terrain quoi et on on se sent démuni (Dirigeant 3, coopérative).

Le souvenir et la narration de cet événement, la « presque » mort symbolique de l'entreprise, décrite de manière détaillée font (ré) émerger les émotions vécues à l'époque. L'émotion survient lors de l'entretien sous forme de pleurs et surprend le narrateur même. Le récit porte sur un événement marquant, le moment où il est devenu dirigeant, et décrit à la fois les difficultés rencontrées (l'échec commercial, les mauvaises relations entre associés) et les émotions associées au vécu des difficultés, énoncées de manière claire et précise : le sentiment de solitude, de difficulté presque insurmontable.

L'extrait présente les conditions dans lesquelles le dirigeant prend la décision de s'imposer comme le directeur, face aux co-gérants. La décision est contraire à l'idéologie de la co-gestion et d'égalité entre les membres de la coopérative, et nous comprenons par l'émergence de ces émotions à quel point enfreindre la règle a été en même temps douloureux et nécessaire pour la " survie » de l'entreprise 
selon le point de vue du dirigeant. Au-delà des réflexions sur l'éventuelle vérité objective des décisions prises, le récit restitue la vérité vécue par le dirigeant et son interprétation du contexte comme moment charnière, marquant la transformation de sa perception à la fois de l'environnement et de son propre rôle. La force et la puissance des émotions accompagnant cette transformation se font papables même des années après les événements.

Dans cet extrait, l'émotion survenue s'associe au fait que le dirigeant a dû prendre une décision contraire aux valeurs du mouvement (la démocratie et l'égalité). L'analyse des émotions ouvre la voie à la compréhension du sens d'être dirigeant et à sa transformation. Ce sens est lié à la difficulté inhérente à l'activité des dirigeants de l'économie sociale : tenir la tension entre les contraintes de la gestion budgétaire et le respect des normes de conduite prônées par le mouvement.

\section{L'identification et la reconnaissance par le sujet lui-même du changement survenu dans le sens du diriger}

Lorsqu'elle a pris son poste dans l'association, cette directrice de 48 ans a reçu des menaces d'une partie de ses collaborateurs. A la fin du récit, elle déclare avoir modifié son état d'esprit au travail comme conséquence de l'événement. Elle attribue la transformation de son attitude à l'événement vécu. Si avant l'événement elle nouait des relations amicales avec ses anciens collègues devenus collaborateurs, elle est par la suite devenue méfiante.

et donc depuis j'ai une attitude euh bon je suis je dirais je suis fréquentable mais je ne sympathise pas voilà (...) je suis très méfiante maintenant ouais (Dirigeante 4 , association).

Euh:: oui oui plusieurs il y a eu deux on va dire je dirais deux moments euh importants deux qui sont euh qui ont fait que ma vie ma vie professionnelle a changé euh:: (...) mais j'avais sûrement besoin de l'entendre ça a fait que je me suis euh j'ai compris j'ai mieux compris qui j'étais comment je me positionnais et comment je devais me positionner ça a changé ma façon un de me positionner d'être et c'est aussi grâce à ça que j'ai pu un jour devenir président d'un directoire (Dirigeant 5 , coopérative).

Dans le deuxième exemple, le dirigeant d'une chaîne de supermarchés coopératifs, reconnaît que l'un des événements marquants, la discussion avec un psychologue, a profondément modifié sa manière de « se positionner ». L'événement détermine un avant et un après dans le regard qu'il porte sur soi et de l'écart entre ce constat et ce qu'il perçoit comme étant attendu dans le milieu professionnel à l'époque. Cet événement est considéré par lui-même comme étant la source d'une transformation du sens : il transforme les liens établis entre sa perception de ses caractéristiques personnelles, ses attentes professionnelles et le milieu dans lequel 
il souhaitait s'insérer à l'époque. Le dirigeant va encore plus loin en argumentant que ce changement a rendu possible son présent de président de directoire et donc de dirigeant. L'argumentation historique, c'est-à-dire le fait d'établir une relation de causalité entre le passé et le présent, semble une attitude spontanée qui résulte $\mathrm{du}$ regard diachronique de sa vie professionnelle. Elle permet au dirigeant de construire et démontrer, dans le cadre de l'entretien, le sens, comme direction, des transformations.

\section{L'énonciation de principes d'action associés à la transformation du sens de diriger}

Dans certains cas, les dirigeants associent à leurs récits des règles de comportement à tenir en tant que dirigeant. Ce sont des règles qui découlent directement de leur expérience et de la transformation du sens attribué à leur activité.

Dans le premier exemple, le dirigeant d'une coopérative d'achat des matériaux pour le bâtiment, glisse dans le récit d'un événement marquant, des règles pour être un «bon directeur de coopérative ». Pour énoncer ces principes, le dirigeant emploie le verbe falloir. Par ce biais, il exprime que l'événement l'a mis face à des circonstances qui l'ont appelé à l'action. En effet, le narrateur se montre obligé à mener ces actions (se poser les bonnes questions; s'interroger sur son propre positionnement vis-à-vis des membres adhérents), qui ne semblent pas spontanées ou confortables pour lui. Le mode " falloir » s'accompagne des pronoms comme « on » qui renforcent le sens de l'injonction à l'action provenant de la situation telle qu'elle a été interprétée.

Parce que bon pendant un moment je rappelais tout le monde j'essaie de savoir qu'est-ce qui se passe il n'y avait pas assez de commandes alors bon donc après on se posait des questions c'est à dire $\underline{\text { il faut toujours se poser les bonnes questions }}$ c'est pas pour dire ć $\underline{c}^{\prime}$ est de leur faute parce que en fait c'est toujours aussi cette tentation que l'on a dans la coopérative ben on vous a mis un truc et vous ne venez pas c'est pas:: ça veut dire que euh c'est jamais l'adhérent qui:: puisque lui il n'a que sa perception individuelle de la coopérative ça veut dire que ce qu'on lui a mis en retour de ce qu'il a apporté ça veut dire qu'on est à côté de la plaque et c'est ça la grande difficulté c'est à dire que:: faut toujours être en adéquation avec ses membres (Dirigeant 6, coopérative).

Ben je suis sur des conversations professionnelles je ne vais pas déjeuner avec le les collaborateurs je ne bois pas le café enfin je veux dire je ne sors pas je suis au boulot avec mes collaborateurs et je ne me mêle pas aux conversations eh eh personnelles eh ou très rarement voilà et je fais toujours très attention d'avoir : euh si c'est des sujets un peu conflictuels d'avoir un tiers je suis rarement en face à face avec quelqu'un ou alors il y a un compte rendu d'entretien à ce moment-là (Dirigeante 4, association). 
Avril Thorne, Kate C. Mc Lean, Amy M. Lawrence (2004) distinguent deux types de sens attribués à des souvenirs : ceux portant sur les "leçons apprises » et ceux portant sur la « compréhension ». Les « leçons apprises » constituent des inférences pratiques tirées de l'expérience et qui, dans un contexte similaire, dirigeraient l'action future. Ces principes de comportement seraient significatifs pour deux raisons : d'une part parce que dans le discours du dirigeant ils sont associés à un événement considéré comme marquant, ce qui définit leur ancrage dans l'expérience réelle du sujet. D'autre part, parce qu'il s'agit de principes pouvant orienter les manières d'évaluer les situations et son propre rôle dans les situations. Ils constitueraient donc des grilles d'analyse de sa manière d'agir dans les situations avec les collaborateurs plutôt que des comportements réels à tenir.

L'énonciation de principes d'action lors de la construction du récit constitue un indicateur de la densité de l'expérience vécue, dans le sens où l'on peut inférer qu'une réflexion approfondie a été menée à l'occasion de l'événement et même après. De cette manière, lorsqu'un narrateur construit un récit d'expérience, celui-ci contient non seulement ce qui est survenu, les faits, mais aussi des éléments relatifs aux processus de réflexion, de construction de sens, inhérent au vécu subjectif des faits. Les principes de comportement exprimeraient une sorte de condensation de l'expérience, mais condensation finalisante car orientée vers l'action souhaitable.

De même que l'événement a donné lieu aux principes, les principes contiennent en eux l'événement, car c'est celui-ci qui leur donne un sens et une légitimité pour le dirigeant. Ces principes ne constituent pas de «bonnes pratiques » dans l'absolu mais seulement au sujet de l'expérience vécue par le dirigeant. Nous faisons alors l'hypothèse qu'ils resteront valides tant que celui-ci ne sera pas démenti soit par un nouvel événement soit par la cumulation d'expériences permettant de les remettre en question.

\section{L'imbrication de la dimension individuelle et de la dimension collective dans les transformations du sens de diriger}

Le dirigeant de cette coopérative a vécu une situation de conflit avec un collaborateur de longue date qui n'a pas respecté les règles déontologiques imposées par la structure.

Les entreprises de l'économie sociale sont aussi des projets politiques et donc quand on est militant euh:: on se raccroche aussi à ça c'est à dire l'entreprise peut aller très mal on se raccroche au aux idées et au projet politique que que l'on construit moi j'ai absolument pas pensé à:: à partir c'est à dire je:: non au contraire il fallait absolument sauver euh euh ce truc-là parce que si coopx avait coulé à ce moment-là les idées politiques portées par coopx auraient pris un coup alors que justement je trouve que depuis euh on arrive à on on est suffisamment forts pour les porter ces idées-là et c'est intéressant (Dirigeant 7, coopérative). 
Être militant de l'économie sociale impose une certaine conduite à tenir. Au moment d'orienter l'action un jeu entre le « je » du dirigeant et le « nous » de l'économie sociale intervient. Ce jeu fait partie des pré-occupations (Clot 2004) accompagnant et orientant la prise de décisions. L'interprétation qui prévaut dans une situation comme celle vécue par ce dirigeant passe de sauver l'entreprise à celle de sauver l'entreprise parce que c'est le moyen de préserver les idées et le modèle de société portées par l'entreprise. La solidarité entre le « je » et le «nous », entre la solitude du dirigeant et le collectif du mouvement de l'économie sociale a un poids déterminant dans le sens construit et dans la transformation de ce sens. Sauver l'entreprise devient ainsi un acte politique et par là même un acte d'affirmation de ses idées. Le collectif devient un levier pour l'action, une force permettant d'endurer la difficulté. Il définit le sens de la transformation, comprise comme ce vers quoi l'entreprise doit tendre.

\section{Conclusion}

Nous avons souhaité analyser dans cet article les éléments structurant les transformations du sens. Quels éléments sont modifiés, du point de vue du sens attribué à l'activité professionnelle, lorsque le sujet fait face à une situation inattendue ?

La transformation dans le temps de ce que diriger une structure de l'économie sociale signifie pour les dirigeants se réalise par un processus mental et émotionnel associant cinq éléments : les liens entre passé, présent et futur ; la présence d'émotions ; l'identification du changement par le sujet lui-même ; le lien avec des principes d'action qui découlent des expériences vécues et l'imbrication de la dimension individuelle et la dimension collective.

Des récits portant sur des événements considérés comme marquants permettent d'identifier les composantes des transformations du sens et de comprendre le sens, en termes de direction, attribué à leur histoire. Ce dernier élément contribue à légitimer, aux yeux du dirigeant, son parcours et l'aboutissement qu'est le poste occupé. Ainsi, la perspective diachronique de l'activité, que nous avons appelé le sens des transformations permet d'identifier la direction imprimée par les dirigeants aux événements successifs qu'ils ont vécus venant expliquer et démontrer ce qu'ils sont devenus à partir de ce qui leur est advenu.

Nous faisons l'hypothèse que la culture propre au mouvement de l'économie sociale donne une coloration toute particulière aux transformations du sens et au sens des transformations. Toutefois, il est pertinent de se poser la question : quelles composantes de la construction de sens se modifient lors que d'autres professionnels vivent des événements marquants ? L'incidence de la dimension culturelle et la nature de l'activité réalisée sur ces transformations reste à être explorée. 
Pour les chercheurs, s'intéresser à la formation professionnelle, au développement de l'activité chez les professionnels dans le temps long d'un parcours, nécessite de se pencher sur des processus parfois presque imperceptibles mais agissant sur les manières de faire et de percevoir son activité. L'analyse des récits d'expérience constituent à notre avis une opportunité de dévoiler ces phénomènes subtils mais non moins importants.

\section{Bibliographie}

Adam J.-M., Revaz F. (1996) L'analyse des récits, Paris, Seuil.

Association pour l'emploi des cadres (2012) Les cadres de l'économie sociale et solidaire, Paris, Apec.

Barbier J.-M. (2017) Vocabulaire d'analyse des activités. Penser les conceptualisations ordinaires, Paris, Presses Universitaires de France.

Barbier J.-M. (2020) Sémantique de l'action et sémantique d'intelligibilité des actions. Le cas de la formation in : Manières de penser et manières d'agir en éducation et en formation, B. Maggi (dir.), Paris, Presses Universitaires de France : 89-104, https://doi.org/10.3917/puf.maggi.2000.01.0089

Barbier J.-M., Chauvigné C., Vitali M.-L. (2011) Diriger : un travail, Paris, L’Harmattan.

Betton E., Cros F., Rodriguez D., Thiévenaz J. (2008) L'activité dirigeants de l'économie sociale, Paris, Cnam, MRPP.

Bruner J. S. (1990) Acts of meaning, London, Harcard University Press.

Bruner J. (1991) The narrative construction of reality, « Critical Inquiry », $\mathrm{n}^{\circ} 18-1: 1-21$, https://www.journals.uchicago.edu/doi/abs/10.1086/448619, https://doi.org/10 $.1086 / 448619$

Clot Y. (2004) Travail et sens du travail in : Ergonomie, P. Falzon (éd.), Paris, Presses Universitaires de France : 317-331, https://doi.org/10.3917/puf.falzo.2004.01.0317

Crossley M. L. (2000) Introducing Narrative Psychology. Self, trauma and the construction of meaning, Buckingham, Open University Press.

Demoustier D. (2001) L'économie sociale et solidaire. S'associer pour entreprendre autrement, Paris, La découverte.

Dewey J. (1993) Logique. La théorie de l'enquête, Paris, Presses Universitaires de France. 
Draperi J.-F. (2009) L'année de l'économie sociale et solidaire 2010 : une alternative à rédecouvrir en temps de crise, Paris, Dunod, https://doi.org/10.3917/dunod.drap .2011 .01

Gendlin E. (1962) Experiencing and the creation of meaning, New York, The free press of Glencoe, https://doi.org/10.1037/t29376-000

Jeantet T. (2004) L'économie sociale en perspective. Rester fidèle aux principes, «Informations Sociales », $\mathrm{n}^{\circ} 116: 58-70$.

Labov W. (1993) Le parler ordinaire. La langue dans les ghettos noirs des Etats-Unis, trad. A. Kihm, Paris, Minuit.

Labov W. (2003) Uncovering event structure of narrative in : Georgetown University Rownd Table on Languages and Linguistics. Linguistics, Language and the Real World: Discourse and Beyond, D. Tannen, J. Alatis (éds.), Georgetown, Georgetown University Press: 63-83.

Labov W. (2010) Oral narratives of personal experiences in : Cambridge Encyclopedia of the Language Sciences, Cambridge, P. Hogan (éd.), Cambridge University Press: 546-548.

Leclerc-Olive M. (1997) Le dire de l'événement (biographique), Paris, Presses Universitaires Septentrion.

Leplat J., Hoc J.-M. (1983) Tâche et activité dans l'analyse psychologique des situations, «Cahiers de Psychologie Cognitive », n 3 : 49-63.

Mintzberg H. (1984) Le manager au quotidien. Les dix rôles du cadre, trad. P. Romelaer Paris, Les éditions d'organisation.

Mintzberg H. (1990) The Manager's job. Folklore and facts, « Harvard Business Review », $\mathrm{n}^{\circ}$ mars-avril : 1-13, https://hbr.org/1990/03/the-managers-job-folklore-and-fact

Mispelblom Beyer F. (2010) Diriger et encadrer l'économie sociale et solidaire : l'exception ou la règle ? in : Management humain des organisations. Grandeurs et misères de la fonction de dirigeant, F. Ben Hassel, B. Raveleau (dir.), Paris, L'Harmattan : 307-322.

Nicolas S. (2005) Comment l'homme conserve-t-il des souvenirs ? in : L'homme cognitif, A. Weil-Barais, Paris, Presses Universitaires de France : 317-430.

Robert V. (2007) L'irrésistible montée de l'économie sociale. Un projet, une culture, des valeurs, Paris, Autrement.

Rodriguez D. (2017) Evènements marquants et (re)construction de sens des dirigeants de l'économie sociale, thèse de doctorat, Paris, Conservatoire national des arts et métiers. 
Rogalski J., Leplat J. (2011) L'expérience professionelle : expériences sédiméntées et expériences épisodiques, "Activités 》, 8 (2) : 4-31, https://doi.org/10.4000/ activites.2556

Sarbin T. R. (1986) Narrative Psychology : The storied nature of human conduct, New York, Praeger.

Throne A., McLean K., Lawrence A. (2004) When remembering is not enough: reflecting on self-defining memories in late adolescence, "Journal of Personality ", $\mathrm{n}^{\circ} 72$ : 513-542, https://doi.org/10.1111/j.0022-3506.2004.00271.x

Tulving E. (1983) Elements of episodic memory, New York, Oxford University Press.

\section{The Meaning of Transformations and Transformations of Meaning: Changes in the Meaning of Activity at the Biographical Scale}

\section{Summary}

This paper discusses the process of transforming the sense of professional activity felt by people managing enterprises in the area of the social economy (insurance companies, associations, cooperatives, foundations). It recognizes the elements that structure these transformations, resulting from significant events. Transformations of the sense of management activity refer to the education process related to preparation for the profession (Rodriguez 2008). The transformation process is triggered by individual/episodic experiences (Rogalski, Leplat 2011), which we identify as significant events.

The sense accompanying the experiences of professional activity is understood here as a process and the result of symbolizing activities expressed in the narrative (Bruner 1991). Meaning transformations are formulated as dynamic articulations of the five elements. They are: the temporal dimension, emotions; the entity noticing the change; formulation of operating principles; interweaving individual and team dimensions. These elements characterize the process of constructing and reconstructing sense from a diachronic perspective.

Our analyses are based on the results of qualitative research conducted with thirteen company managers in free-form interviews. As a result of the research, we obtained 39 narratives relating to those situations in the professional sphere in which the narrators had a sense of collision with a completely new and unexpected situation.

Managers of enterprises in the area of social economy are especially privileged to analyse the transformation of the meaning of their activity, because their activities belong to the movement of ideas and economic initiatives with a strongly coloured by the values they defend (solidarity, responsibility, commitment...). This forces them 
to act in two ways: facilitating the activity of their co-workers by showing them the direction of action, and inscribing meaning and their actions into the ideas of the movement.

\section{Sens transformacji i transformacje sensu: zmiany sensu aktywności w wymiarze biograficznym}

\section{Streszczenie}

W artykule omawiany jest proces transformacji sensu aktywności profesjonalnej odczuwanego przez osoby kierujące przedsiębiorstwami z obszaru ekonomii społecznej (towarzystwa ubezpieczeniowe, stowarzyszenia, spółdzielnie, fundacje). Dokonano w nim rozpoznania elementów strukturyzujących te transformacje, powstałe w efekcie zdarzeń znaczących. Transformacje sensu aktywności kierowania odnoszą się do procesu kształcenia związanego z przygotowaniem do zawodu (Rodriguez 2008). Proces transformacji uruchamiają doświadczenia jednostkowe/epizodyczne (Rogalski, Leplat 2011), które zidentyfikowaliśmy jako zdarzenia znaczące.

Sens towarzyszący doświadczeniom aktywności profesjonalnej ujmowany jest tu jako proces i wypadkowa aktywności symbolizacyjnych, wyrażanych w narracji (Bruner 1991). Transformacje sensu są formułowane jako dynamiczne artykulacje pięciu elementów. Są nimi: wymiar temporalny, emocje; dostrzeżenie zmiany przez podmiot; sformułowanie zasad działania; przeplatanie wymiarów indywidualnych i zespołowych. Te elementy charakteryzują proces konstruowania i rekonstruowania sensu w perspektywie diachronicznej.

Nasze analizy są oparte na wynikach badań jakościowych przeprowadzonych $\mathrm{z}$ trzynastoma kierownikami przedsiębiorstw, w formie wywiadów swobodnych. W rezultacie badań otrzymaliśmy 39 narracji odnoszących się to tych sytuacji w sferze zawodowej, w których narratorzy mieli poczucie zderzenia z sytuacją zupełnie nową i nieoczekiwaną.

Kierujący przedsiębiorstwami z obszaru ekonomii społecznej są szczególnie uprzywilejowani do analizowania transformacji sensu podejmowanej aktywności, ponieważ ich działania należą do ruchu idei i inicjatyw ekonomicznych o silnym zabarwieniu wartościującym, które je tworzą (solidarność, odpowiedzialność, zaangażowanie...). Co zmusza ich do podwójnego postępowania: ułatwianie aktywności współpracownikom poprzez wskazywanie im kierunku działania oraz wpisywanie sensu i ich działań w idee tego ruchu.

\section{Pour citer cet article}

Rodriguez D. (2021) Le sens des transformations et les transformations du sens : les changements du sens de l'activité à l'échelle biographique, "Nauki o Wychowaniu. Studia Interdyscyplinarne" 2(13), 167-185, https://doi.org/10.18778/24504491.13 .11 